\title{
Adrenal Hemorrhage as a Rare Cause of Neonatal Anemia Associated with Hydrocephalus Secondary to Intraventricular Hemorrhage-A Case Report ${ }^{*}$
}

\author{
Krushnakumar Kesan\#, Rahul Kumar Gupta, Paras Kothari, Abhaya Gupta, Ritesh Ranjan, \\ Kedar Mudkhedkar, Parag Karkera \\ MCh Pediatric Surgery, Department of Pediatric Surgery, LTMMC \& LTMGH Sion, Mumbai, India. \\ Email: "krishnakesan@rediffmail.com
}

Received May $8^{\text {th }}, 2013$; revised June $10^{\text {th }}, 2013$; accepted July $13^{\text {th }}, 2013$

Copyright (C) 2013 Krushnakumar Kesan et al. This is an open access article distributed under the Creative Commons Attribution License, which permits unrestricted use, distribution, and reproduction in any medium, provided the original work is properly cited.

\begin{abstract}
Neonatal adrenal hemorrhage is frequently associated with birth trauma, perinatal asphyxia, intrauterine infection, coagulation defects and thromboembolism. It has varied clinical presentation depending on degree of hemorrhage and amount of adrenal cortex compromised by hemorrhage. The most common clinical presentations are persistent jaundice and flank mass. We report a case of left sided adrenal hemorrhage in a breech delivered male neonate with perinatal asphyxia presented with anemia and fever. On further evaluation, he was also having moderate communicating hydrocephalus secondary to intraventricular hemorrhage. The adrenal hemorrhage was managed conservatively. Subsequent abdominal ultrasound showed resolving adrenal hemorrhage. Right ventriculoperitoneal shunt was done for hydrocephalus. Postoperative course was uneventful. The patient is asymptomatic at follow-up.
\end{abstract}

Keywords: Adrenal Hemorrhage; Neonatal Anemia; Hydrocephalus; Intraventricular Hemorrhage

\section{Introduction}

The adrenal gland in neonatal age is vulnerable to hemorrhage because of its large size and high vascularity [1]. The incidence of adrenal hemorrhage in detected cases ranges from 1.7 to 2.1 per 1000 births. Because adrenal bleeding may remain asymptomatic, the real occurrence is probably higher [2]. Neonatal adrenal hemorrhage is frequently associated with birth trauma, perinatal asphyxia, intrauterine infection, coagulation defects and thromboembolism [1,3]. Adrenal hemorrhage usually presents with neonatal jaundice, paleness and/or flank mass, discoloration of the scrotum, anemia and adrenal insufficiency [4-6]. In this report, we present a rare case of neonatal adrenal hemorrhage having anemia and hydrocephalus.

\section{Case Report}

A 25-day-old, $2.8 \mathrm{~kg}$, male neonate was referred to us with complaint of fever and severe anaemia. Records revealed that he was born of nonconsanguineous mar-

${ }^{*}$ Conflict of interest: NIL.

${ }^{\#}$ Corresponding author. riage, fullterm, breech delivered with history of delayed cry. APGAR scores were 4 and 5 at 1 and 5 minutes, respectively. Patient was admitted to intensive care unit for perinatal asphyxia.

On general examination gross pallor was evident and there was no icterus. An abdominal mass was palpated in the left flank. In addition, the baby was having wide open anterior fontanels.

Haematological investigations were suggestive microcytic hypochromic anaemia $(\mathrm{Hb} 6.2 \mathrm{gm} \%$, Platelet count $7.1 \mathrm{lac} / \mathrm{cu} \mathrm{mm})$. Renal function and liver function tests including coagulation profile were normal. Blood culture and urine culture were sterile. TORCHS' test [toxoplasmosis, other infections, rubella, cytomegalovirus (CMV), and herpes simplex virus (HSV)] was also negative.

Abdominal ultrasound showed a well defined hypoechoic lesion in left suprarenal area, of size $4 \times 3 \mathrm{~cm}$, at the upper pole of the left kidney suggestive of adrenal mass which could be either neuroblastoma or adrenal hemorrhage. The margins between the mass and the left kidney were indistinct. Contrast enhanced computerised tomography (CECT) abdomen revealed a $3 \times 3.5 \times 4 \mathrm{~cm}$ 
sized well defined cystic density lesion with enhancing walls in left paravertebral region suggestive of adrenal hemorrhage with superadded infection (Figures 1(a) and (b)). USG and CT skull revealed moderate dilatation of all four ventricles. CT also revealed intraventricular hemorrhage in right temporoparital region (Figure 2).

Serum cortisol level and 24 - hour urinary vanillyl mandelic acid (VMA) levels were normal, which ruled

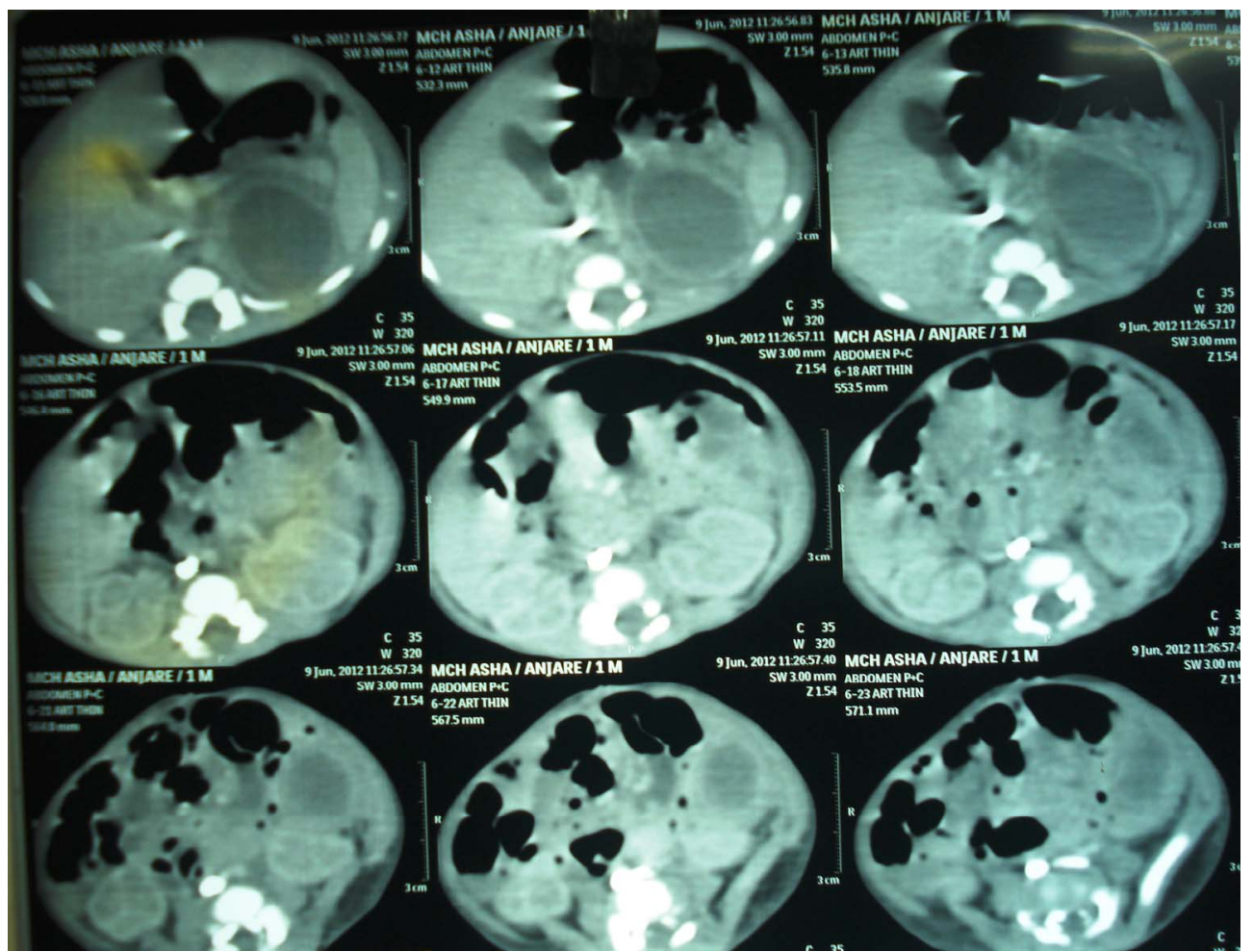

(a)

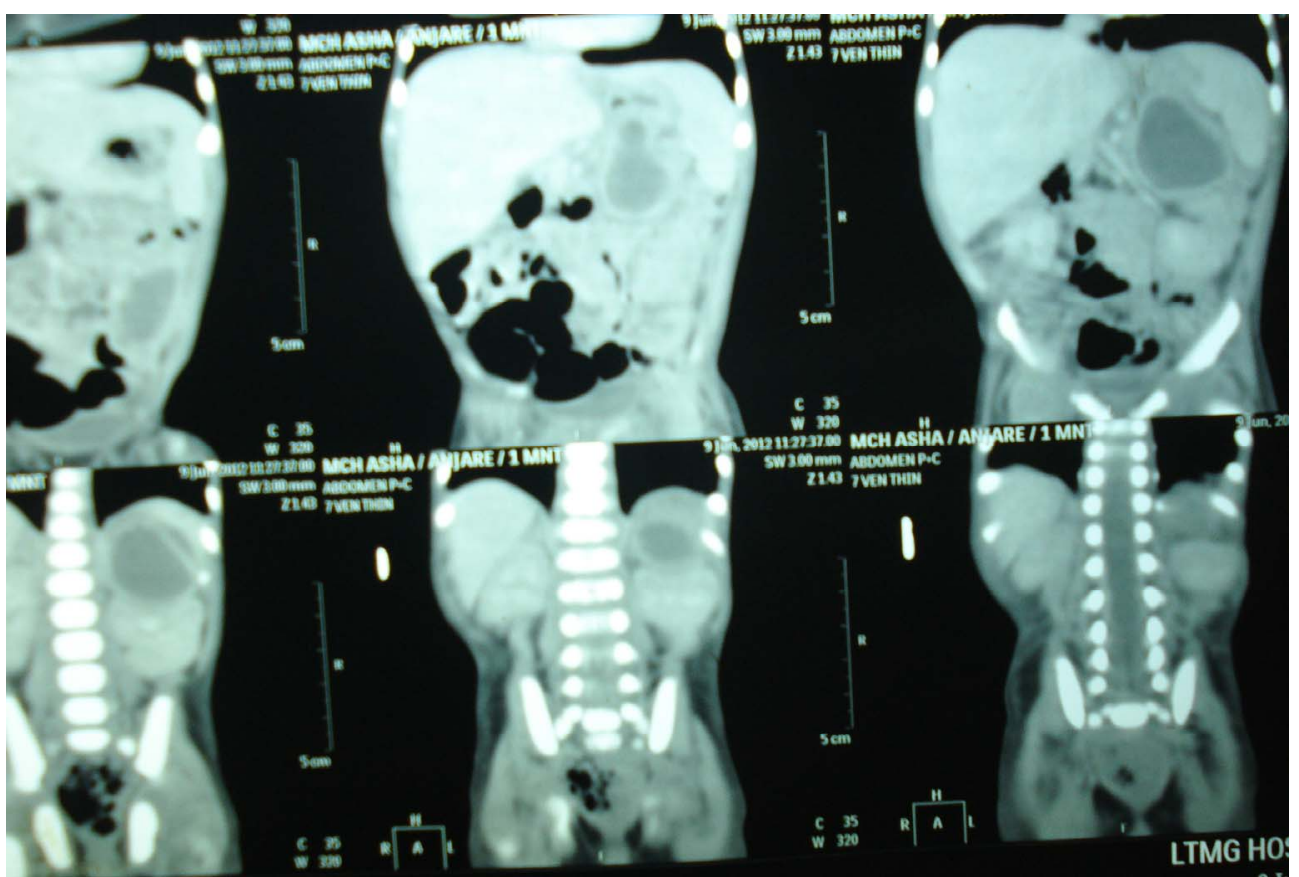

(b)

Figure 1. CECT abdomen showing well defined cystic density lesion with enhancing walls in left paravertebral region suggestive of adrenal hemorrhage. 


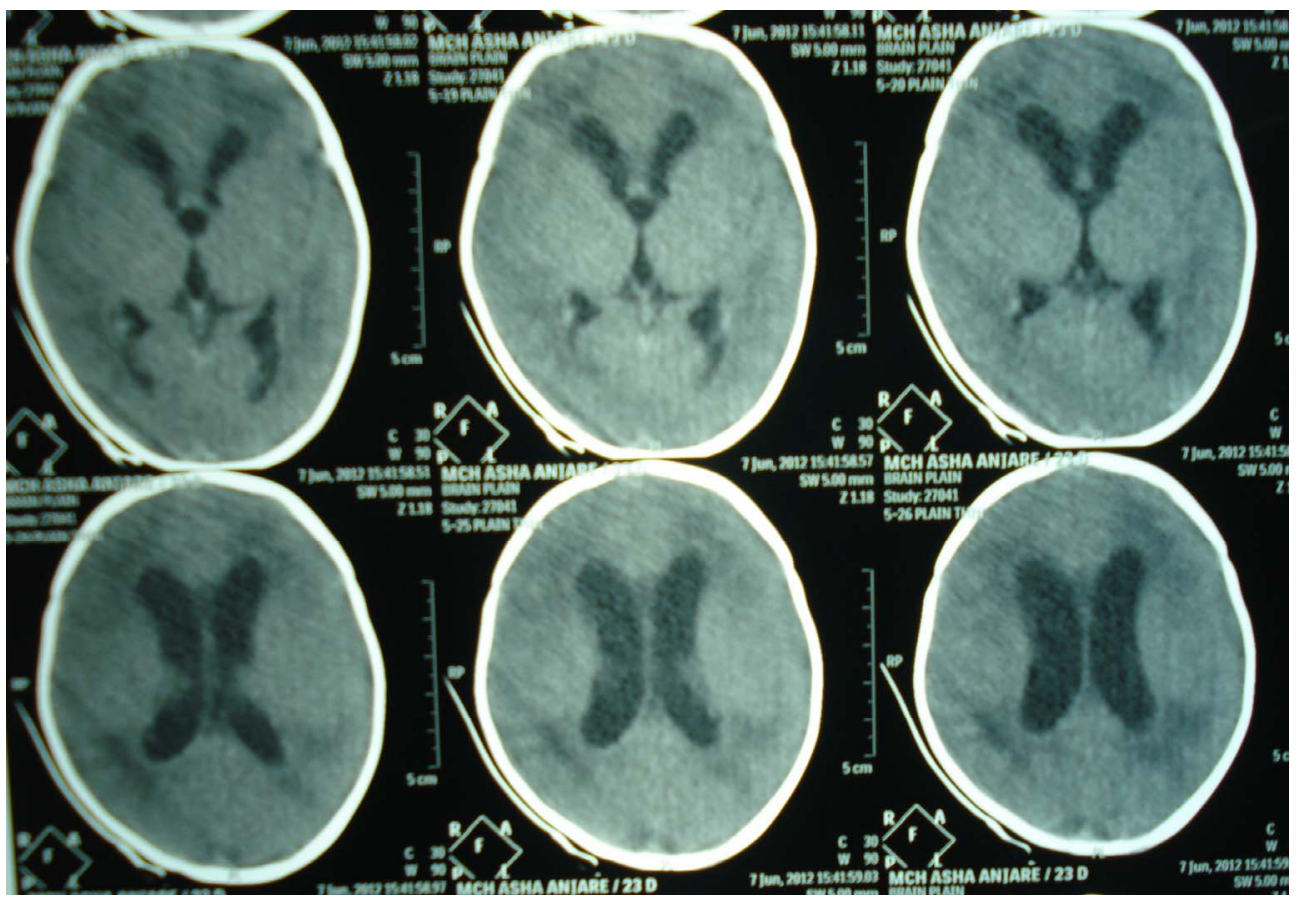

Figure 2. CECT abdomen showing well defined cystic density lesion with enhancing walls in left paravertebral region suggestive of adrenal hemorrhage.

out neuroblatoma as a differential diagnosis.

The patient was initially managed with blood transfusions and intravenous antibiotic. Adrenal haemorrhage was managed conservatively. Subsequent serial abdominal ultrasound done at 1 week interval for 2 weeks showed gradual reduction in size of adrenal hemorrhage. Ventricular CSF was tapped showing plenty of RBC. Patient was kept on oral acetazolamide and CSF tap repeated. Right ventriculoperitoneal shunt was done for hydrocephalus when CSF was clear. Postoperative course was uneventful. The patient is asymptomatic at 6 months of follow up and USG at 6 months showed complete resolution of hemorrhage.

\section{Discussion}

Adrenal hemorrhage is a relatively uncommon condition frequently seen in term male infants delivered vaginally $[7,8]$. The incidence of detected cases ranges from 1.7 to 2.1 per 1000 births [4]. Adrenal hemorrhage is more common in neonates than in children or adults because of large size (relative to body weight gland is 10 to 20 times larger than adults) and unique blood supply. It is supplied by subcapsular plexus formed by fifty to sixty arterial branches from three suprarenal arteries. This plexus is drained into medullary sinusoids via a very few venous channels. In case of hypoxia due to any cause there occurs shunting of blood from vital organ to adrenal gland which resulting into hemorrhage. Various predisposing factors are birth trauma, perinatal asphyxia, intrauterine infection, coagulation defects and thromboembolism, or it can be spontaneous.

There is a male predominance for this condition. Right side is more commonly affected than left and 5\% - 10\% cases may be bilateral [9]. Right adrenal gland is more susceptible, as it is more likely to be compressed between the liver and spine and, the right adrenal vein usually drains directly into the inferior vena cava, so it is prone to changes in venous pressure.

Adrenal hemorrhage may be asymptomatic or may presents with neonatal jaundice, paleness and/or flank mass, discoloration of the scrotum, anemia and adrenal insufficiency [4]. Clinical presentations depend on degree of hemorrhage and amount of adrenal cortex compromised by hemorrhage. Our patient was having anemia with flank mass, in addition moderate communicating hydrocephalus and intraventricular hemorrhage.

In case of neonatal adrenal hemorrhage it is very important to rule out neuroblastoma particularly the cystic form. 24-hour urinary excretion of VMA should be done, since an increase in VMA is virtually diagnostic of neuroblastoma [10]. Other differential diagnosis of $\mathrm{AH}$ are neonatal adrenal abscess, cortical renal cyst, obstructed upper cortical renal cyst and an obstructed upper excretory tract in the duplicated kidney [11].

Diagnosis is established with USG and CECT abdomen. Serial abdominal USG are more helpful as they 
show resolving adrenal hemorrhage. The pattern of echogenicity of an adrenal hematoma depends on its age. An early-stage hematoma appears solid with diffuse or inhomogeneous echogenicity. As liquefaction occurs, the mass demonstrates mixed echogenicity with a central hypoechoic region and eventually becomes completely anechoic and cyst like. Calcification can appear as early as 1 - 2 weeks after hemorrhage.

Presentation of adrenal hemorrhage with neonatal anemia, flank mass has been reported by number of authors $[2,12]$. But association between adrenal hemorrhage and hydrocephalus has not been reported in literature. Both conditions can be attributed with common etiological factor of perinatal asphyxia.

\section{Conclusion}

In case of neonatal anaemia with perinatal asphyxia, adrenal haemorrhage should be suspected. Most of adrenal haemorrhage resolves with conservative. Our patient was having adrenal hemorrhage and hydrocephalus secondary to intraventricular hemorrhage.

\section{REFERENCES}

[1] M. Rumińska, J. Welc-Dobies, M. Lange, et al., "Adrenal Haemorrhage in Neonates: Risk Factors and Diagnostic and Clinical Procedure," Medycyna Wieku Rozwojowego, Vol. 12, No. 1, 2008, pp. 457-462.

[2] N. Demirel, A. Y. Baş, A. Zenciroğlu, et al., "Adrenal Bleeding in Neonates: Report of 37 Cases," The Turkish Journal of Pediatrics, Vol. 53, No. 1, 2011, pp. 43-47.

[3] A. Vulkova, K. Kovacheva, M. Ionov, et al., "Adrenal Hemorrhage in a Newborn with Factor V Leiden: A Clinical Case," Akusrsko Ginekologiceskoe (Sofiia), Vol. 48, No. 5, 2009, pp. 47-50.

[4] M. Mutlu, G. Karagüzel, Y. Aslan, et al., "Adrenal Hemorrhage in Newborns: A Retrospective Study," World
Journal of Pediatrics, Vol. 7, No. 4, 2011, pp. 355-357. http://dx.doi.org/10.1007/s12519-011-0259-7

[5] U. A. Qureshi, N. Ahmad, A. Rasool, et al., "Neonatal Adrenal Hemorrhage Presenting as Late Onset Neonatal Jaundice," Journal of Indian Association of Pediatric Surgeons, Vol. 14, No. 4, 2009, pp. 221-223. http://dx.doi.org/10.4103/0971-9261.59607

[6] A. T. Abdu, V. M. Kriss, H. S. Bada, et al., "Adrenal Hemorrhage in a Newborn," American Journal of Perinatology, Vol. 26, No. 8, 2009, pp. 553-557. http://dx.doi.org/10.1055/s-0029-1214239

[7] S. C. Velaphi and J. M. Perlman, "Neonatal Adrenal Hemorrhage: Clinical and Abdominal Sonographic Findings," Clinical Pediatrics, Vol. 40, 2001, pp. 545-548. http://dx.doi.org/10.1177/000992280104001002

[8] V. Miele, M. Galluzzo, G. Patti, G. Mazzoni, et al., "Scrotal Hematoma Due to Neonatal Adrenal Hemorrhage: The Value of Ultrasonography in Avoiding Unnecessary Surgery," Pediatric Radiology, Vol. 27, No. 8, 1997, pp. 672-674. http://dx.doi.org/10.1007/s002470050209

[9] M. Tuchman, M. L. Ramnaraine, W. G. Woods, et al., "Three Years of Experience with Random Urinary Homovanillic and Vanillylmandelic Acid Levels in the Diagnosis of Neuroblastoma," Pediatrics, Vol. 79, 1987, pp. 203-205.

[10] M. Pery, J. K. Kaftori and J. A. Bar-Maor, "Sonography for Diagnosis and Follow Up of Neonatal Adrenal Hemorrhage," Journal of Clinical Ultrasound, Vol. 9, No. 7, 1981, pp. 397-340. http://dx.doi.org/10.1002/jcu.1870090708

[11] G. Bergami, S. Malena, M. Di Mario, et al., "Echography in the Follow-Up of Neonatal AH. The Presentation of 14 Cases," Radiologia Medica, Vol. 79, 1990, pp. 474-478

[12] M. A. Akin, D. Coban, S. Doganay, et al., "Intrahepatic and Adrenal Hemorrhage as a Rare Cause of Neonatal Anemia," Journal of Perinatal Medicine, Vol. 39, No. 3, 2011, pp. 353-354. http://dx.doi.org/10.1515/jpm.2011.018 